\title{
Community-acquired Klebsiella pneumoniae central nervous infection with acute suppurative otitis and bacteremia: a case report in mainland China
}

\section{Ruixue Sun}

Peking Union Medical College Hospital

Hui Zhang

Peking Union Medical College Hospital

Yingchun Xu

Peking Union Medical College Hospital

Huadong Zhu

Peking Union Medical College Hospital

\section{Xuezhong Yu}

Peking Union Medical College Hospital

Jun Xu ( $\nabla$ pumchxujun@126.com )

Peking Union Medical College Hospital https://orcid.org/0000-0001-7967-8743

\section{Case report}

Keywords: Klebsiella pneumoniae, meningitis, brain abscesses, acute suppurative otitis, case report

Posted Date: January 22nd, 2020

DOI: https://doi.org/10.21203/rs.2.21621/v1

License: (1) This work is licensed under a Creative Commons Attribution 4.0 International License. Read Full License 


\section{Abstract}

Community-acquiredKlebsiella pneumoniae (K. pneumoniae) central nervous system (CNS) infection combined with bacteremia was rarely identified world. We received a 55-year-old womanwho was under hormone therapy because of Sjogren's syndrome.The onset of this case was acute suppurative otitis,then she developed headache and unconscious, the cerebrospinal fluid (CSF) and imaging examinations were compatible with K. pneumoniae meningitis and suspicious brain abscesses, and K. pneumoniae bacteremia was also combined. Even with adequate antibiotic and supportive therapy, the patient' relatives refused further assessment of intracranial lesions and took the patient back home, finally the patient died of no spontaneous breathing. Hormone therapy might be the risk factor of communityacquiredK. pneumoniaeinfection. Appropriate antibiotic application and infection focus drainage should be tried for treating this condition. The reason of patient's death might be the CNS infection combined with K. pneumoniaebacteremia and the absence of intracranial lesion elimination. This case is reported in order to help us know this rare disease and promote a further progress on its pathogenesis and treatment.

\section{Introduction}

Klebsiella pneumoniae (K. pneumoniae), which is a gram-negative aerobic rod-shaped bacterium, is usually hospital-acquired and occur primarily in patients with impaired host defenses [1]. Communityacquired infection caused by K. pneumoniae mainly reported in cases of invasive liver abscess syndrome (ILAS) of liver abscess, meningitis, or endophthalmitis in Taiwan [2]. Although sporadic cases occur otherwhere in the world recently, community-acquired K. pneumoniae central nervous infection without liver abscess are rarely seen worldwide [3-11]. Here, we reported our experience with a failed case of an adult with community-acquired K. pneumoniae meningitis associated with acute suppurative otitis, bacteremia and suspicious multiple brain abscesses.

\section{Case Presatation}

A 55-year-old woman from Hebei province with Sjogren's syndrome for 2 years was taking methylprednisolone $24 \mathrm{mg} / \mathrm{d}$ orally. Her right ear discharged pus 8 days ago, and soon she developed a fever and headache, then unconscious followed 6 days later. On examination, the patient's vital signs were stable, but she had coma with glasgow coma scale score $1+1+3$. She appeared nuchal rigidity and positive right Babinski sign, other signs were negative.

White blood count was $10.92 \times 109 /$ I with an elevated neutrophil ratio of $89.0 \%$, hemoglobin $11.1 \mathrm{~g} / \mathrm{dl}$ and platelet count $302 \times 109 / \mathrm{l}$. The CRP was $>160 \mathrm{mg} / \mathrm{L}$, live and kidney function was normal.PCT $4.5 \mathrm{ng} / \mathrm{ml}$. Lumbar puncture yielded pale yellow cloudy CSF with the pressure of $>330 \mathrm{mmH}_{2} \mathrm{O}$, and its characteristics were as follow: WBC count, $1147 \times 106 / \mathrm{L}$, with a predominance of polymorphonuclear leukocytes; total protein, $2.48 \mathrm{~g} / \mathrm{L}$; and glucose, $<0.11 \mathrm{mmol} / \mathrm{L}, \mathrm{Cl}, 109 \mathrm{mmonl} / \mathrm{L}$. Head and temporal CT scan revealed right mastoiditis, abdominal B ultrasound results were normal. The patient was diagnosed as purulent meningitis and right mastoiditis besides Sjogren's syndrome and was treated by cefatriaxone 
$2 \mathrm{~g} \mathrm{q12h}$ and vancomycin $1 \mathrm{~g} \mathrm{q12h}$, mannitol helped reduce intracranial pressure. The patient's consciousness didn't recover and was transferred to EICU at the same day.

On hospital day 2, K. pneumoniae grew in a culture of the CSF and blood and was sensitive to all the tested antibiotics including amikacin, ceftazidime, ceftriaxone, cefotaxime, cefepime, estapamil, meropenem, meropenem, simvastatin, cefoperazone / sulbactam and piperacillin / tazobactam. The antibiotic was adjusted to be meropenem $2 \mathrm{~g}$ q8h and amikacin $0.4 \mathrm{~g} \mathrm{q} 12 \mathrm{~h}$, the dose of methylprednisolone was reduced to $10 \mathrm{mg} / \mathrm{d}$ gradually to help control infection. However, the patient's condition aggravated, and trachea cannula was used for airway protection.

Because of the continuous fever and unconscious, CT scan and lumbar puncture were reviewed on hospital day 7 , the contrast-enhanced head CT indicated new-onset multiple lamellar low-density umbra on bilateral frontal, parietal, occipital and right temporal lobes without abnormal enhancement (Finger 1 patient's contrastenhanced head CT). The pressure of CSF decreased to $220 \mathrm{mmH}_{2} \mathrm{O}$, the WBC count in CSF reduced to $163 \times 106 / \mathrm{L}$, the protein was $3.55 \mathrm{~g} / \mathrm{L}$. We planned to order contrast-enhanced head MRI and ask the neurosurgery for clear the infection focus. However, the patient's relatives refused further treatment due to the possible poor prognosis. Sadly, the patient died on her way back home because of no spontaneous breathing.

\section{Discussion}

Cases of community-acquired K. pneumoniae meningitis are rare observed in the mainland of China, the only patient reported was associated with ILAS. Our patient might be the first one presented with community-acquired K. pneumoniae central nervous infection and bacteremia [12]. Unfortunately, the patient died in the end, so here we reviewed the related literatures about communityacquired K. pneumoniae meningitis and try to analyze the reason of our patients' terrible prognosis.

8 patients with community-acquired K. pneumoniae meningitis which was not associated with liver abscess were reported in Caribbean, Singapore, Italy, UK, Taiwan and US [3,4,9,13-15]. But all of them didn't have brain abscess or bacteremia. Two of them had uncontrolled dabetes and another two had chronic alcoholic diseases, in this case, hormone therapy may explain the patient's increased risk of developing meningitis with K. pneumoniae instead of the the common.

The primary infection source could be the suppurative otitis, which is the similar with 4 patients reported with preceding infections of endophthalmitis, otitis and sphenoid sinusitis. However, K. pneumoniae was also cultured in the patient's blood, and the low-density umbra on CT scan seemed to be multiple brain abscesses. This is the first patient showed K. pneumoniae bacteremia associated with meningitis, and as the direct spread of organisms from a contiguous site usually causes a single brain abscess $[18,19]$, the disseminated brain abscesses in this case may be hematogenous spread by bacteremia instead of direct spread by suppurative otitis. But the common conditions leading to hematogenous seeding of the brain usually include chronic pulmonary infections, skin infections, pelvic and intraabdominal infections, 
bacterial endocarditis and esophageal dilation and endoscopic sclerosis of esophageal varices, So, the pathogenesis of this case is still unclear, and CNS infection combined with bacteremia could be the risk factor of patient's bad prognosis.

Besides supportive treatment, the main therapy of patients with K. pneumoniae meningitis was timely antibiotics. The 7 surviving patients with $\mathrm{K}$. pneumoniae meningitis were treated by the three-generation cephalosporin combined with pefloxacin or gentamicin or amikacin, and only one case used meropenem $[3,4,9,13-15]$, but the sensitivity results were not provided. In our cases, K. pneumoniae cultured from the CSF were susceptible to all of the remaining antibiotics tested, meropenem and amikacin were choosed based on the sensitivity in vitro, unfortunately, the ending was still tragic. Infection focus elimination is also the key point, one surviving patient with endophthalmitis was performed rans pars plana vitrectomy, and another patient with inoperable infected pancreatic pseudocyst died treated with reasonable antibiotics. Because of our patient's relatives giving up, the patient didn't have chance of estimating and clearing the intracranial lesion, this was the other reason of patient's tragedy.

What's more, we also suspect that K. pneumoniae which leading to community-acquired CNS infection and bacteremia has any special virulence factors. as we known, K. pneumoniae strains related to ILAS harbors capsular serotype K1 or K2 which are more virulent than that with non-K1/K2. Besides capsular serotype, hypermucoviscosity phenotype, lipopolysaccharide, siderophores, and pili also contribute to the infection pathogenesis of K. pneumoniae $[1,2]$. Therefore, our further study direction will be the analysis on virulence factors of $\mathrm{K}$. pneumoniae leading to community-acquired meningitis without ILAS.

In conclusion, community-acquired K. pneumoniae CNS infection without ILAS was rarely identified worldwide. Hormone therapy might be the risk factor of infection. Appropriate antibiotic application and infection focus drainage should be tried for treating this condition. The reason of patient's death might be the combination with K. pneumoniae bacteremia, the absence of estimating and clearing the intracranial lesion and unknown special but stronger virulence factors of $\mathrm{K}$. pneumoniae. This case is reported in order to help us know this rare disease and promote a further progress on its pathogenesis and treatment. In this way, patients with this situation will gain a better prognosis.

\section{Declarations}

\section{Statement}

The patient's next to kin has given their consent to publish the patients' cases for this study.

\section{Ethics approval and consent to participate}

The Institutional Review Board (IRB) of Peking Union Medical College Hospital has reviewed the study and has determined that this is a retrospective study and the design is scientifically and is up to the ethics standards. The IRB thus approve the study.

\section{Consent for publication}


Consent for publication has been obtained from the patients reported in this article.

\section{Availability of data and materials}

The datasets used and analyzed during the current study are available from the corresponding author on reasonable request.

\section{Competing interests}

The authors declare that they have no conflicts of interests.

\section{Funding}

No funding

\section{Acknowledgements}

Not applicable

\section{References}

[1] Weill Cornell Medicine.Recent advances in the understanding and management of Klebsiella pneumoniae. F1000Res. 2017 Sep 27; 6:1760.

[2] Fang CT, Lai SY, Yi WC, et al. Klebsiella pneumoniae genotype K1: an emerging pathogen that causes septic ocular or central nervous system complications from pyogenic liver abscess. Clin Infect Dis. 2007;45(3):284.

[3] Bénédicte Melot, Sylvain Brisse, Sébastien Breurec, et al. Community-acquired meningitis caused by a CG86 hypervirulent Klebsiella pneumoniae strain: first case report in the Caribbean. BMC Infectious Diseases. 2016; 16:736.

[4] Habib AG, Tambyah PA.Community-Acquired Klebsiella pneumoniae Central Nervous System Infections in Adults in Singapore. Eur J Clin Microbiol Infect Dis. 2003; Aug;22(8):486-8.

[5] Lucio Boglione, Claudia Spezia, Filippo Lipani, et al. Klebsiella pneumoniae meningitis in a 38-year-old Chinese traveller with impaired glucose tolerance: A new emerging syndrome? Travel Medicine and Infectious Disease. 2008; 6: 32-35.

[6] Felipe F Tuon, Jaime L Rocha, Lavinia N Arend, et al. Treatment and outcome of nine cases of KPCproducing Klebsiella pneumoniae meningitis. The British Infection Association. 2013; 4:161-164.

[7] Yanagawa T, Nakamura H, Takei I, et al. Klebsiella pneumoniae meningitis associated with liver abscess: a case report. Jpn J Antibiot. 1989 Oct;42(10):2135-40. [8] Ohmori S, Shiraki K, Ito K, et al. Septic 
endophthalmitis and meningitis associated with Klebsiella pneumoniae liver abscess. Hepatol Res. 2002; Apr 22(4):307-312.

[9] Mario Giobbia, Pier Giorgio Scotton, Antonella Carniato, et al. Community-acquired KZebsieUa pneumoniae bacteremia with meningitis and endophthalmitis in Italy. Int J Infect Dis. 2003; 7: 234-235.

[10] Hsin-I Shih, Hsin-Chun Lee, chiao-hsiung chuang, et al. Fatal Klebsiella pneumoniae and Emphysematous Brain Abscess After Endoscopic Variceal Ligation in a Patient with Liver Cirrhosis and Diabetes Mellitus. J Formos Med Assoc. 2006; 105: 857-860.

[11] Michael Saccente. Klebsiella pneumoniae Liver Abscess, Endophthalmitis, and Meningitis in a Man with Newly Recognized Diabetes Mellitus. Clinical Infectious Diseases. 1999; 29:1570-71.

[12] Yun Qian, Chi-Chun Wong, San-Chuan Lai, et al. Klebsiella pneumoniae invasive liver abscess syndrome with purulent meningitis and septic shock: A case from mainland China. World J Gastroenterol. 2016; March 7; 22(9): 2861-2866.

[13] Orzechowska A, Lacey S, Soosay G, et al.Community-acquired Klebsiella pneumoniae meningitis in an alcoholic patient with an infected pancreatic pseudocyst; a case report and review of literature. $J$ Med Case Rep. 2007 Oct 29; 1:116

[14] Liao SB, Yang KJ, Lai CC, et al. Bilateral endogenous Klebsiella pneumoniae endophthalmitis associated with meningitis-useful vision regained after treatment: case report. Chang Gung Med J. 2000 Sep;23(9):566-71.

[15] Bianca Lee, Kevin Yeroushalm, Hay Me Me, et al. Community acquired Klebsiella pneumoniae meningitis: a case report. Germs. 2018 Jun 4;8(2):92-95.

\section{Figures}



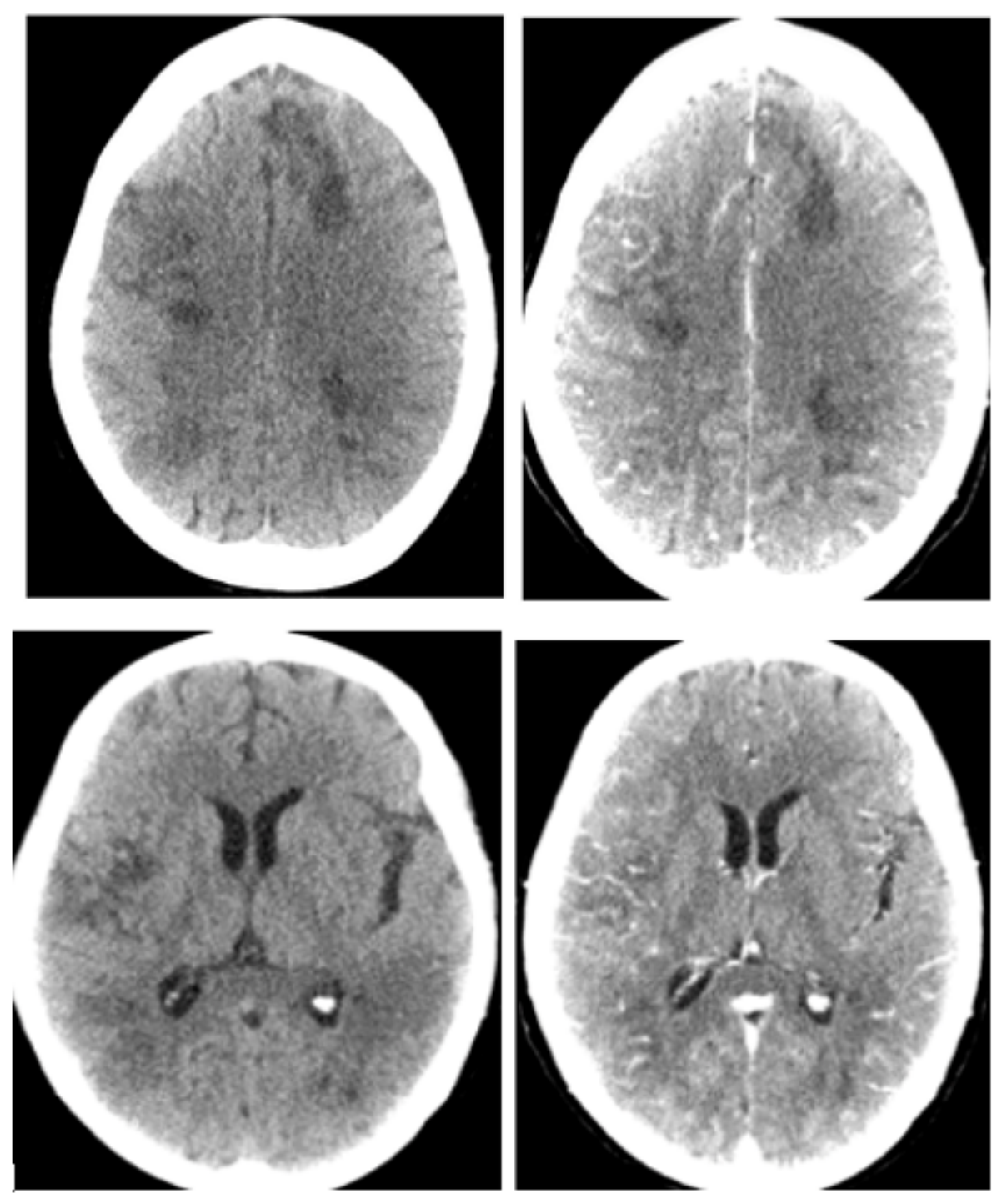

Figure 1

multiple lamellar low-density umbra on bilateral frontal, parietal, occipital and right temporal lobes without abnormal enhancement 